\begin{tabular}{|c|c|}
\hline Title & Double network hy drogels based on semi-rigid polyelectrolyte physical networks \\
\hline Author(s) & Takahashi, Riku; Ikai, Takuma; Kurokawa, Takayuki; King, Daniel Rudolf; Gong, Jian Ping \\
\hline Citation & $\begin{array}{l}\text { Journal of materials chemistry B, Materials for biology and medicine, } 7(41), 6347-6354 \\
\text { https://doi.org/10.1039/C9TB01217F }\end{array}$ \\
\hline Issue Date & 2020-11-09 \\
\hline Doc URL & http:/hdl.handle.net/2115/79727 \\
\hline Type & article (author version) \\
\hline File Information & JMaterChemB(2019)_Takahashi-Ikai.pdf \\
\hline
\end{tabular}

Instructions for use 
Journal of Materials Chemistry $B$

Materials for biology and medicine

\section{Accepted Manuscript}

This article can be cited before page numbers have been issued, to do this please use: R. Takahashi, T. Ikai, T. Kurokawa, D. R. King and J. P. Gong, J. Mater. Chem. B, 2019, DOI: 10.1039/C9TB01217F.
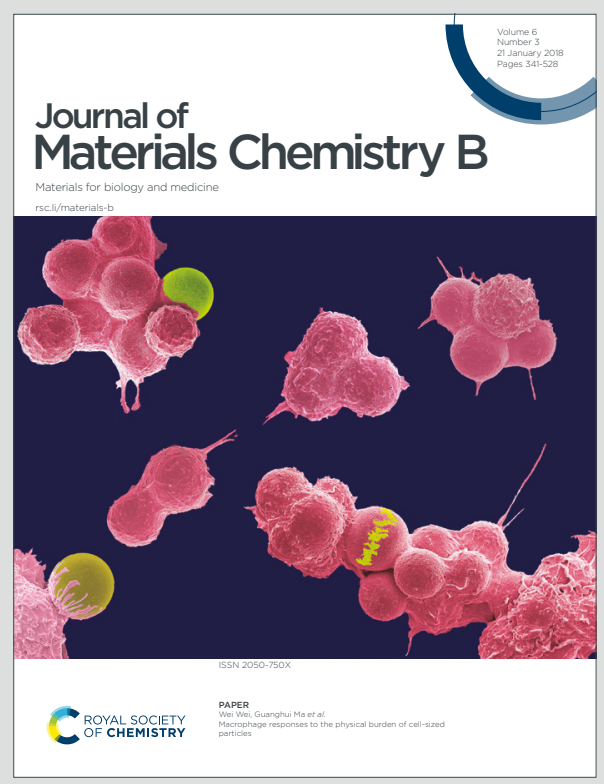

This is an Accepted Manuscript, which has been through the Royal Society of Chemistry peer review process and has been accepted for publication.

Accepted Manuscripts are published online shortly after acceptance, before technical editing, formatting and proof reading. Using this free service, authors can make their results available to the community, in citable form, before we publish the edited article. We will replace this Accepted Manuscript with the edited and formatted Advance Article as soon as it is available.

You can find more information about Accepted Manuscripts in the Information for Authors.

Please note that technical editing may introduce minor changes to the text and/or graphics, which may alter content. The journal's standard Terms \& Conditions and the Ethical guidelines still apply. In no event shall the Royal Society of Chemistry be held responsible for any errors or omissions in this Accepted Manuscript or any consequences arising from the use of any information it contains. 


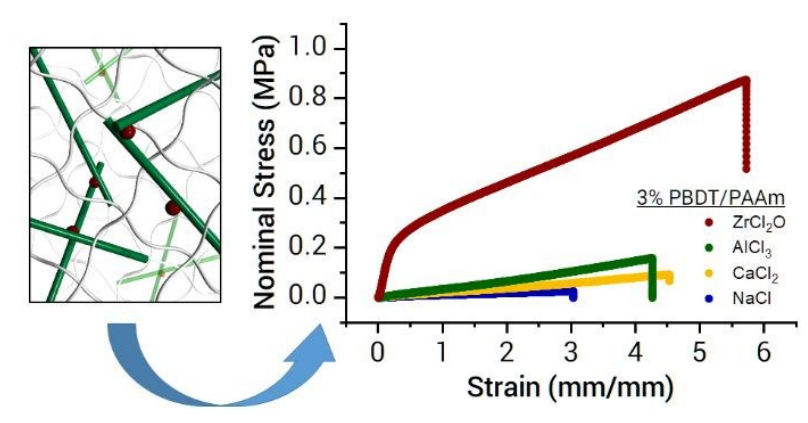

Double network hydrogels are formulated by from polyacrylamide hydrogels containing semi-rigid polyelectrolytes through post-polymerization immersion in multi-valent ion solutions. 


\title{
Double Network Hydrogels Based on Semi-rigid Polyelectrolyte Physical Networitis 177
}

Riku Takahashi ${ }^{1, \$, \dagger}$, Takuma Ikai ${ }^{1, \dagger}$, Takayuki Kurokawa ${ }^{2,3}$, Daniel R. King ${ }^{2,3, *}$ Jian Ping Gong ${ }^{2,3,4 *}$

${ }^{1}$ Graduate School of Life Science, Hokkaido University, Sapporo 001-0021, Japan.

${ }^{2}$ Faculty of Advanced Life Science, Hokkaido University, Sapporo 001-0021, Japan.

${ }^{3}$ Global Station for Soft Matter, Global Institution for Collaborative Research and Education (GI-

CoRE), Hokkaido University, Japan.

${ }^{4}$ Institute for Chemical Reaction Design and Discovery (WPI-ICReDD), Hokkaido University, Sapporo, 001-0021, Japan.

\$Current Address: NTT Basic Research Laboratories, NTT Corporation, 3-1 Morinosato-Wakamiya, Atsugi, Kanagawa, 243-0198, Japan.

$\dagger$ The authors contributed equally to this work

*Corresponding author

KEYWORDS: Hydrogels, Semi-rigid polymers, Composite materials, Double network gels, Superstructures, tunable mechanical properties,

\begin{abstract}
Applying the double network principle to develop tough hydrogels with different polymer chemistries is important for the potential application of hydrogel materials. Synthesis of the two interpenetrated networks with contrasting structure and properties required for double networks usually involves a two-step polymerization process. In this work, we present a new method to synthesize tough double network hydrogels by post-physical crosslinking of linear semi-rigid polyelectrolytes entrapped in a chemically crosslinked neutral network. Owing to their semi-rigid structure, the linear polyelectrolytes form a brittle physical network above their overlap concentration in multi-valent $\mathrm{ZrCl}_{2} \mathrm{O}$ ion solutions without macroscopic phase separation within the flexible neutral network. The double network hydrogels thus prepared exhibit high modulus $(\sim 1.7 \mathrm{MPa})$, strength $(\sim 1.3 \mathrm{MPa})$, fracture strain $(\sim 7.3)$, and strain energy density $\left(\sim 5.9 \mathrm{MJ} / \mathrm{m}^{3}\right)$, while containing over $80 \%$ water. These materials also exhibit modest self-healing ability ( $\sim 51 \%$ after 30 minutes), demonstrating an additional benefit of a physical sacrificial network. This method is simpler than the conventional two-step polymerization and could be applied to develop tough hydrogels from rigid polyelectrolytes, including biopolymers such as DNA, HA, and chondroitin sulfate.
\end{abstract}


Hydrogels, a class of soft/wet materials, exhibit unique properties that cannot be found in hard/dry materials, including low friction ${ }^{1}$, high biocompatibility $^{2}$, and high permeability ${ }^{3}$. Conventional hydrogels are brittle and fragile however, limiting their applications. The brittleness of hydrogels originates from heterogeneity in the network structure and high solvent content. ${ }^{4,5}$ The former induces stress concentration locally during deformation, and the latter causes low resistance against crack propagation. To overcome such limitations, intense efforts have focused on developing mechanisms to increase the mechanical properties of hydrogels. Breakthroughs have occurred owing to the development of the following techniques: 1) suppressing crack initiation by homogenization of the network structure (e.g. slide-ring gels and Tetra-PEG gels) ${ }^{6,7}$, and 2) preventing crack propagation by stress yielding (nanocomposite gels and double network (DN) gels). ${ }^{8,9}$ Among them, DN gels, consisting of an interpenetrating brittle/hard network and stretchable/soft network, exhibit noteworthy properties including high Young's modulus $\left(\sim 10^{5}-10^{6} \mathrm{~Pa}\right)$ and tremendous toughness $\left(\sim 4500 \mathrm{~J} / \mathrm{m}^{2}\right)$ despite containing high $(\sim 90 \%)$ water content. ${ }^{10,11}$ Therefore, DN gels have great potential for applications in biomedical fields that required stiffness, toughness, and biocompatibility. ${ }^{12,13}$

The profound toughness of DN gels is due to the "sacrificial bond principle" 11 , where energy is dissipated by fracture of the brittle/hard network prior to rupture of the stretchable/soft network. Conventionally, we synthesize a chemically crosslinked polyelectrolyte network as the sacrificial network. Polyelectrolytes are chosen because they exhibit a high swelling ratio in pure water. When soaked in a monomer solution of the second stretchable/soft network, the crosslinked polyelectrolyte network extends, taking up large quantities of solution even with a relatively high crosslinking density, which is subsequently polymerized. This results in a double network gel consisting of a highly extended polyelectrolyte first network at low concentrations interpenetrated with a dense and stretchable second network. Due to this fabrication process, a two-step polymerization is necessary to obtain typical DN gels with contrasting interpenetrated network structure, requiring much time and effort. Additionally, since the first network is chemically crosslinked, it is not capable of exhibiting 


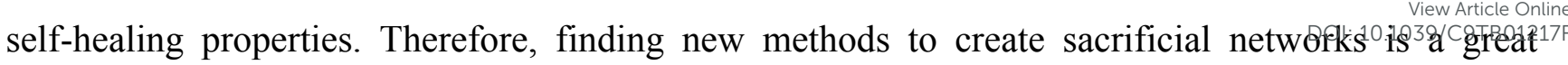
challenge for not only improving the simplicity of the conventional method but also obtaining outstanding soft materials for use in aqueous environments with enhanced mechanical properties.

To achieve these goals, we designed a technique to fabricate physical/chemical DN hydrogels through a "one-pot" polymerization, based on post-ion crosslinking of linear rigid polyelectrolytes entrapped in a chemically crosslinked neutral network (Figure 1). We choose intrinsically rigid polyelectrolytes because 1) the rigid rod structure greatly contrasts the flexible neutral polymer, which should improve the stiffness of the composite gel, 2) the high persistence length should allow for overlap to occur at small concentrations, and 3) the rigid structure prevents collapse into a condensed phase in high ionic strength environments, which occurs for flexible polyelectrolytes.

To demonstrate this idea, we combined the semi-rigid polyelectrolyte, poly(2,2'-disulfonyl-4,4'benzidine terephthalamide) (PBDT) and a chemically crosslinked polyacrylamide network. PBDT has a long persistence length of $\sim 40 \mathrm{~nm}$ to give a small overlap concentration $\left(\mathrm{C}^{*} \sim 0.3 \mathrm{wt} \%\right)^{14}$. PBDT is water soluble, and has found use in applications ranging from viscosity modifiers to gel electrolytes. ${ }^{15-}$ ${ }^{17}$ PBDT and other polyelectrolytes have shown the ability to form a hard/brittle physically crosslinked network by ion complexation with multi-valent ions. ${ }^{18-21}$ To synthesis the DN gels by a one-pot process, this polyelectrolyte is added to a precursor solution containing acrylamide and a chemical crosslinker, and subsequently a PAAm chemically crosslinked gel containing linear PBDT molecules is polymerized. After that, the PBDT-containing PAAm gel is immersed in multi-valent ion solution to form a physical network of PBDT. By this physical gel formation, PBDT/PAAm DN gels consisting of a brittle/hard sacrificial PBDT network and the stretchable polyacrylamide network is formed. Notably, the formation of the sacrificial physical network occurs after the synthesis of the stretchable network, in contrast to traditional DN gels. Unique properties including high dissipation and modest self-healing of the sacrificial network were observed. We found that the multi-valent $\mathrm{ZrCl}_{2} \mathrm{O}$ ions act as a unique crosslinker for the formation of the physical PBDT network, which forms above the overlap concentration of PBDT. The method introduced here enables the fabrication of physical/chemical DN 


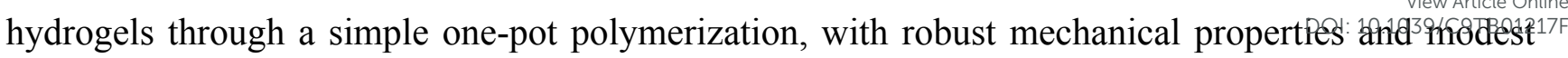
self-healing abilities. This method may be applicable for fabricating DN gels from rigid polymers including biopolymers such as DNA, HA, and chitosan.

\section{a Semi-Rigid Polyelectrolyte}

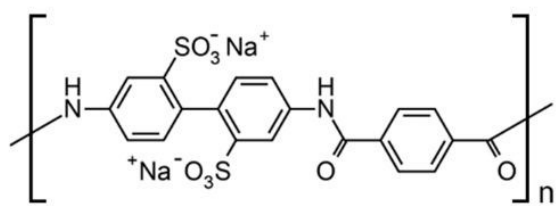



Flexible Neutral Polymer<smiles>CCC(C)C(C)C(N)=O</smiles>
PAAm

Figure 1. Fabrication concept for one-pot DN hydrogels utilizing a semi-rigid polymer-based physical sacrificial network. a) Chemical structures of the semi-rigid polyelectrolyte, PBDT, and flexible neutral polymer, polyacrylamide (PAAm). b) (i) Schematic illustration of the semi-rigid polymer network formed inside the stretchable polymer network via immersion in multi-valent ion solutions. The persistence length of PBDT is $\sim 100$ times greater than PAAm, and therefore is depicted as rod-like for illustration purposes. (ii) Crosslinking of the sacrificial network occurs by physical bonding between the negatively charged PBDT and positively charged ions. The green chains, grey chains and red spheres represent the PBDT, PAAm and multi-valent ions, respectively.

\section{RESULTS AND DISCUSSION}

\section{Characterization of PBDT/PAAm Hydrogels}

Initial characterization of the system was carried out on samples containing $3.0 \mathrm{M}$ PAAm and $3 \mathrm{wt} \%$ PBDT in the as-prepared state. To verify the formation of the PBDT network inside the PAAm hydrogel matrix, we compared the swelling ratio $(\mathrm{Q}$, equilibrium volume divided by the as-prepared volume $)$ of PBDT/PAAm gels and neat PAAm gels in solutions containing various types of ions $(0.15$ M) (Figure 2a and Figure 2b). The neat PAAm gels showed almost the same swelling ratio $(\mathrm{Q}=4 \sim 5)$, regardless of solution. On the other hand, the swelling ratio of the PBDT/PAAm gels were strongly related to the solution. Due to the high osmotic pressure of the polyelectrolyte, the PBDT/PAAm gels exhibited high swelling in pure water $(\mathrm{Q} \sim 9.5)$, which is known as the molecular stent effect. ${ }^{22}$ In the 
$\mathrm{NaCl}$ solution, the swelling ratio of PBDT/PAAm gels is much lower than that in pureplitef still slightly higher than that of pure PAAm gels, indicating that the $0.15 \mathrm{M}$ monovalent metal ions can somewhat suppress the osmotic pressure of the polyelectrolytes, but not completely. However, in the $\mathrm{CaCl}_{2}$ and $\mathrm{AlCl}_{3}$ solutions, the swelling ratios of the PBDT/PAAm gels were slightly less than that of the PAAm gels. When the PBDT/PAAm gels were immersed in the $\mathrm{ZrCl}_{2} \mathrm{O}$ solution, the swelling ratio decreased dramatically $(\mathrm{Q} \sim 2)$, likely due to the PBDT molecules forming a network structure as shown in Figure 1b(i). $\mathrm{ZrCl}_{2} \mathrm{O}$ exists as a complex highly hydrated compound,

$\mathbf{a}$

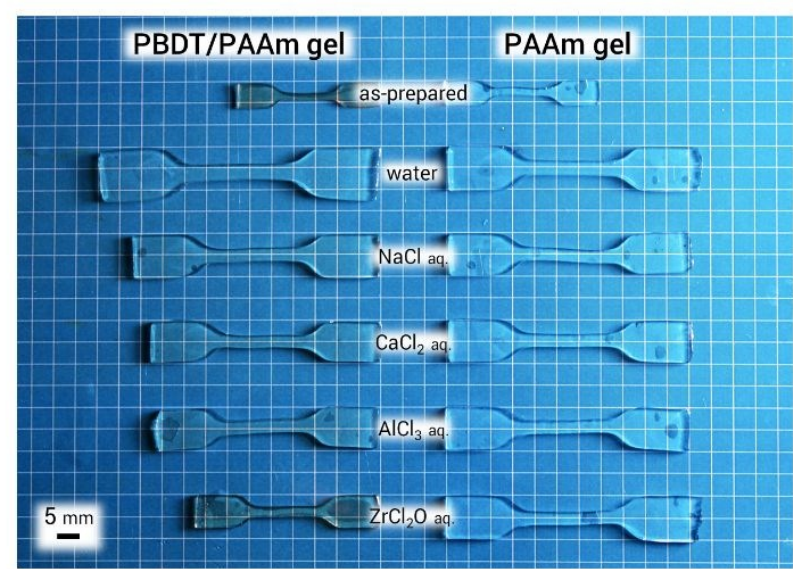

b

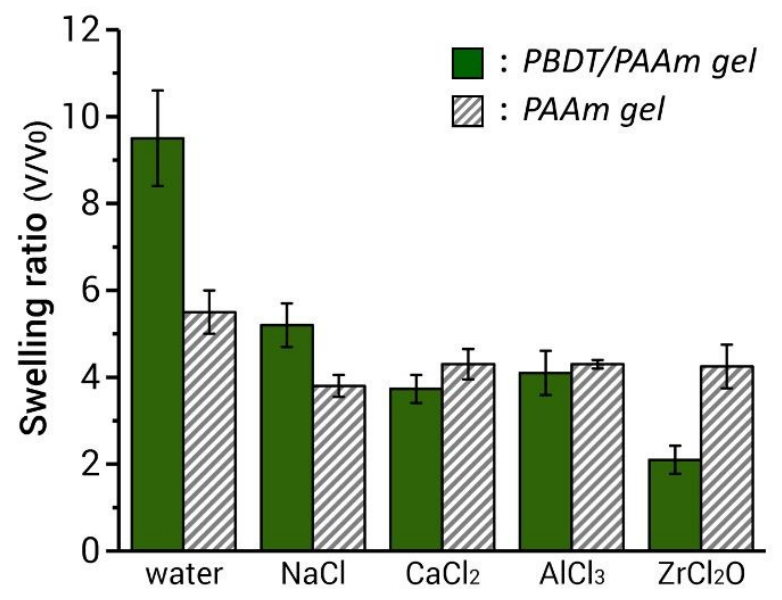

C

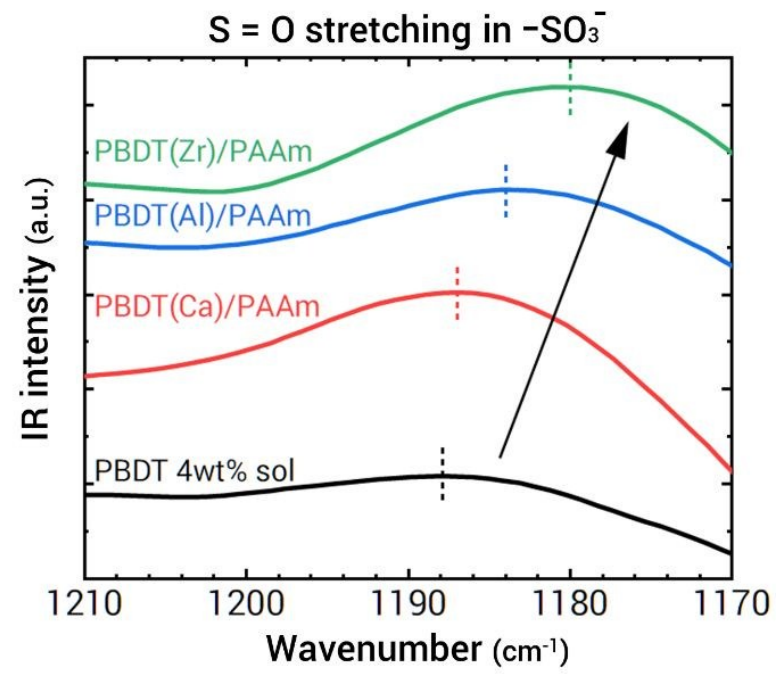

d

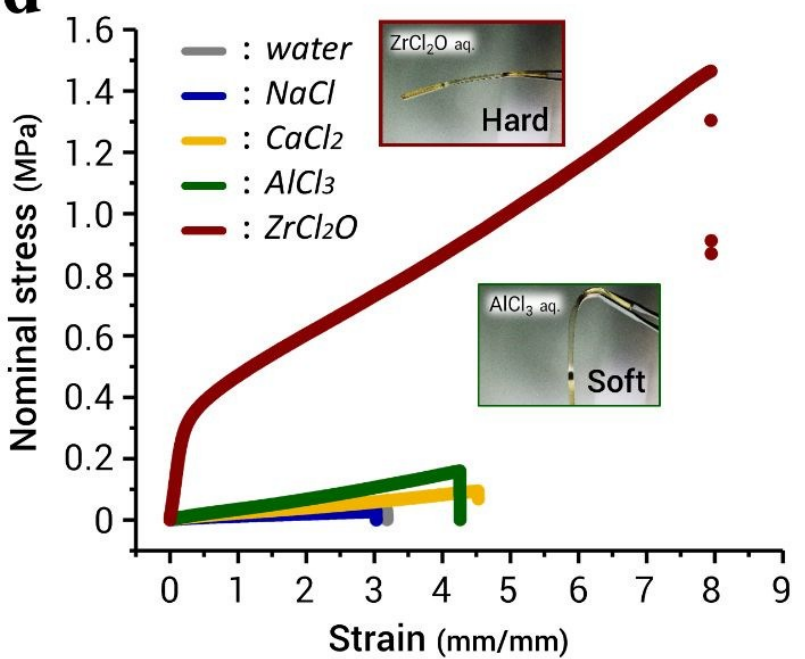

Figure 2. Physical and mechanical properties of PBDT/PAAm gels. a) Photographs of 3 wt\% PBDT/PAAm and neat PAAm gels in the asprepared state and swollen state in various ion solutions. b) Swelling ratio of the gels after immersion in different ion solutions. c) ATRFTIR experiments were performed on PBDT physical gels with varying ion type. Gels were made containing 4 wt\% PBDT and ionic concentration of $0.15 \mathrm{M}$. Peak shifts were compared to PBDT $4 \mathrm{wt} \%$ solutions in water. A vertical shift was applied to the data for clarity. and d) stress-strain curves of $3 \mathrm{wt} \%$ PBDT/PAAm gels formed in different ion solutions with ionic concentration of $0.15 \mathrm{M}$. Insets demonstrate the qualitative difference in strength between the $\mathrm{ZrCl}_{2} \mathrm{O}$ and $\mathrm{AlCl}_{3}$ immersed samples. 


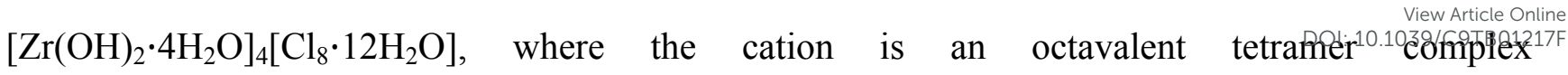
$\left[\mathrm{Zr}(\mathrm{OH})_{2} \cdot 4 \mathrm{H}_{2} \mathrm{O}\right]_{4}{ }^{8+} \cdot{ }^{23}$ We will refer to this structure as $\mathrm{ZrCl}_{2} \mathrm{O}$ for simplicity.

Physical network formation by ionic bonding was verified by attenuated total reflectance Fourier-transform infrared spectroscopy (ATR-FTIR, Figure 2c). A 4 wt $\%$ solution of PBDT in water was prepared. This solution was dropped into $0.15 \mathrm{M}$ solutions containing $\mathrm{CaCl}_{2}, \mathrm{AlCl}_{3}$, or $\mathrm{ZrCl}_{2} \mathrm{O}$. The resulting physical gel was then placed on the ATR crystal and analyzed. A characteristic peak for the $\mathrm{S}=\mathrm{O}$ stretching of the sulfonate group was observed at $1187 \mathrm{~cm}^{-1}$ for the $4 \mathrm{wt} \%$ PBDT aqueous solutions in the un-crosslinked form. After immersing the $4 \mathrm{wt} \%$ PBDT solution into $0.15 \mathrm{M}$ ion solutions, the sulfonate peak shifted to lower wavenumber with increasing valency of the ions. We hypothesize that the strongest network structures can form with $\mathrm{ZrCl}_{2} \mathrm{O}$ because the compound is octavalent (+8), meaning it can form many bonds with the sulfonate groups of PBDT. Since immersion in $\mathrm{ZrCl}_{2} \mathrm{O}$ solution causes the gel to exhibit the largest shift in the FTIR spectrum, we can infer that this gel has the highest concentration of physical bonds. This agrees with the swelling results. The decreased swelling ratio observed in PBDT/PAAm gels with high valency cations likely occurs because PBDT molecules can strongly bind and form physical networks in the PAAm matrix, suppressing osmotic swelling.

The formation of the PBDT network in the PAAm gel matrix caused a significant increase in mechanical properties, similar to the increase seen in traditional double network hydrogels. Figure 2d shows typical tensile stress-strain curves of the $3 \mathrm{wt} \%$ PBDT/PAAm gels swollen in various $0.15 \mathrm{M}$ solutions. The water-equilibrated PBDT/PAAm gel was soft and elastic with low Young's modulus of $14 \pm 0.2 \mathrm{kPa}$, low fracture stress of $26 \pm 3 \mathrm{kPa}$, and low fracture strain of $2.8 \pm 0.4$. No ions are present to crosslink the sacrificial network, and therefore it has no influence on the mechanical response of the gel. In the case of the $\mathrm{NaCl}$ solution-equilibrated sample, the gels exhibited similar mechanical properties to the water-swollen sample. Monovalent ions cannot act as a crosslinking agent, only forming associations with individual chains. The $\mathrm{CaCl}_{2}$ and $\mathrm{AlCl}_{3}$ solution-swollen samples were also soft and elastic; however, the mechanical properties did increase slightly with the increasing cationic 


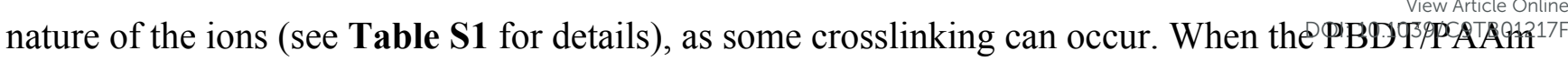
gels were immersed in $\mathrm{ZrCl}_{2} \mathrm{O}$ solution, a clear yielding behavior with high Young's modulus of 1.69 $\pm 0.02 \mathrm{MPa}$, high fracture stress of $1.34 \pm 0.09 \mathrm{MPa}$, and high fracture strain of $7.29 \pm 0.47$ was observed. This dramatic increase in mechanical properties coincides well with the result of the swelling ratio of the PBDT/PAAm gels, which implies the formation of a sacrificial network.

\section{Effect of $\mathrm{ZrCl}_{2} \mathrm{O}$ Concentration on Mechanical Properties}

As the cation valency increases, the number of associations that can occur also increases. Modifying the number of associations is analogous to changing the crosslink density of the first network in chemical DN gels, which is important towards DN gel mechanical characteristics. ${ }^{24}$ In DN gels containing a physically crosslinked first network, ionic concentration also influences physical crosslinking density (Figure 3). Figure 3a contains stress-strain curves for $3 \mathrm{wt} \%$ PBDT/PAAm gels in varying concentration $\mathrm{ZrCl}_{2} \mathrm{O}$ solutions. Figure 3b, Figure 3c, and Figure 3d contain the Young's modulus, fracture strain, and fracture stress from these stress-strain curves, respectively. We see in general that mechanical properties exhibit a parabolic shape with increasing $\mathrm{ZrCl}_{2} \mathrm{O}$ concentration. At low concentrations, the gel is weaker and more fragile, exhibiting properties more closely related to
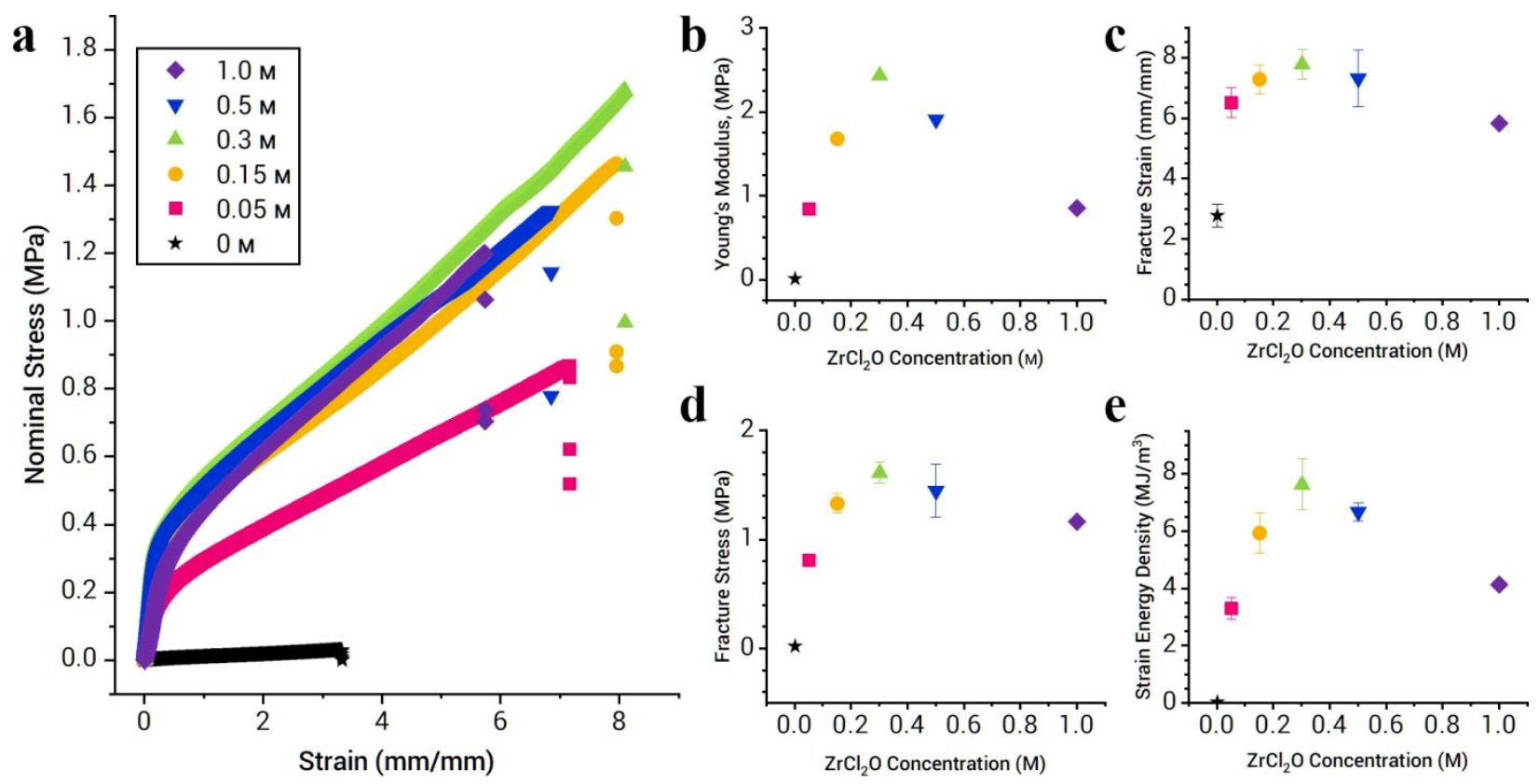

Figure 3. Mechanical properties of the 3\% PBDT/PAAm gels immersed in $\mathrm{ZrCl}_{2} \mathrm{O}$ solutions from 0 to $1 \mathrm{M}$. a) Stress-strain curves. b) Young's modulus. c) Strain at break. d) Stress at break. e) Strain energy density. The error bars are standard deviation from the results of 3-5 samples and are present unless the error bars are smaller than the symbol size. 


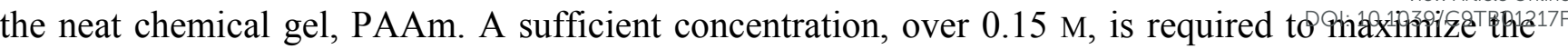
number of crosslinking events; one $\mathrm{ZrCl}_{2} \mathrm{O}$ ion should form physical bonds with more than one PBDT chain. Concentration that is too high suppresses the probability for a multi-valent ion to form ionic bonding to multiple chains. If excess ions are added, above $0.5 \mathrm{M}$, the sulfonate groups on PBDT may become saturated, and not all cations of the $\mathrm{ZrCl}_{2} \mathrm{O}$ complex will bind with other PBDT molecules. If some $\mathrm{ZrCl}_{2} \mathrm{O}$ remains unbound, more swelling will occur and a weaker gel will result. We find that between $0.15 \mathrm{M}$ and $0.50 \mathrm{M}$, the mechanical properties become optimized. This is clearly seen from the strain energy density, a measure of toughness calculated by integrating the area under the stressstrain curve, Figure 3e. With a concentration of $0.3 \mathrm{M}$, the strain energy density reaches $7.6 \pm 0.9$ $\mathrm{MJ} / \mathrm{m}^{3}$. This value is comparable to the toughest double network and viscoelastic hybrid gels. By optimizing the ion type and concentration, very tough hydrogels can be fabricated based on a physical sacrificial network.

\section{Healing Ability}

Many tough hydrogels have been fabricated that utilize ionic interactions as a healing mechanism. ${ }^{25,26}$ When stress is released, elastic restoring force causes recovery of strain and ionic interactions can reform. In this system we observed partial self-recovery. Cyclic tests were performed by applying either low ( $\varepsilon=0.5$, Figure 4a) or high $(\varepsilon=4$, Figure $4 \mathbf{b})$ strains at a constant displacement rate, and then returning to zero strain at the same displacement rate. Recoverability was measured as a function of waiting times between cycles. During the first cycle of the virgin sample, significant hysteresis is observed. This is evidence of the destruction of the sacrificial network; an important property of DN gels is hysteresis during cycle testing. However, in contrast to DN gels, upon reloading the samples, for both low and high strains, some hysteresis is recovered. Hysteresis recovery as a function of waiting time is shown for low and high strain in Figure 4c and Figure 4d, respectively. In both cases, we see that as waiting time increases, healing efficiency increases. Maximum healing efficiencies were measured for low strain of $51.3 \%$ after 30 minutes, and for high strain of $23.8 \%$ after 

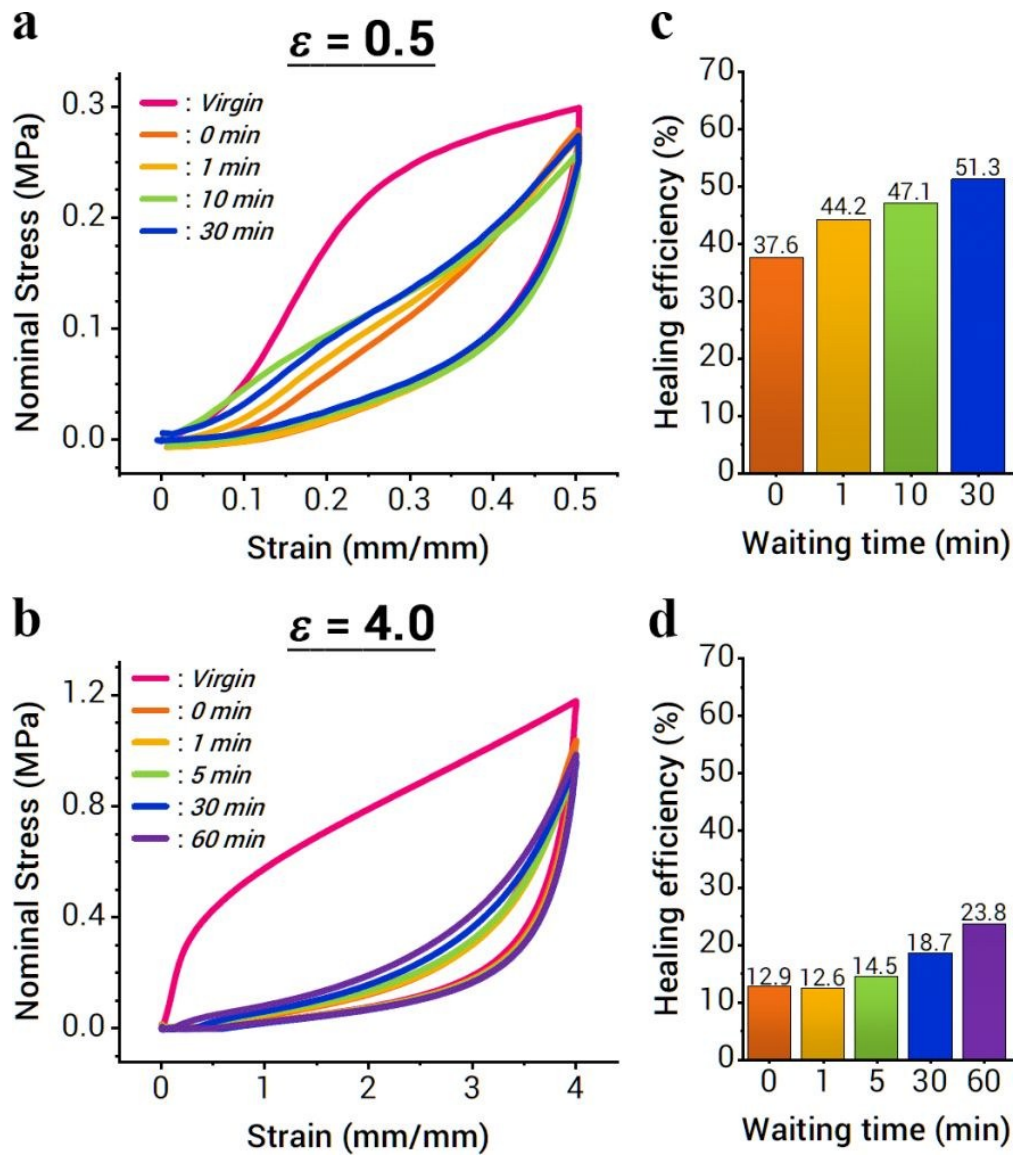

Figure 4. Cyclic loading / unloading test of the 3\% PBDT/PAAm gels after equilibration in $0.15 \mathrm{M} \mathrm{ZrCl}_{2} \mathrm{O}$ solution. Stress-strain curves for cycle testing at strains of (a) 0.5 or (b) 4.0 with different waiting times after testing. Healing efficiency at a maximum strain of (c) 0.5 and (d) 4.0 was estimated by comparing the mechanical hysteresis of the first cycle test to the cycle tests after various waiting times.

one hour. These results provide additional evidence that energy is dissipated through the physical sacrificial network via dynamic interactions. ${ }^{27}$ The toughening mechanism of PBDT/PAAm gels shares many similarities with recently reported stiff and tough gels such as phase-separated PAAm gels ${ }^{28}$ and tri-block copolymer DN gels, ${ }^{29}$ where breaking physical bonds is the primary mechanism of energy dissipation. A significant benefit of utilizing physical bonds in the sacrificial network is their ability to heal after removing the applied deformation.

\section{Effect of PBDT Concentration on Sacrificial Network Formation}

To form a double network structure, the PBDT molecules must exist in sufficiently high concentrations to form a continuous network structure by ionic crosslinking. Next, we aim to elucidate the influence of PBDT concentration on the structure formation and resulting properties of the physical DN gel. As we saw in Figure 2a, the presence of a sacrificial network resists swelling. Samples were 


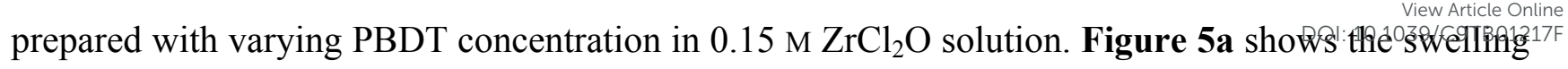




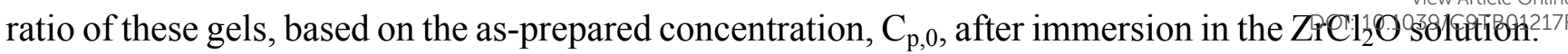
We see that all samples show swelling relative to the as-prepared state $(\mathrm{Q}>1)$, which indicates that the equilibrium concentration of PBDT is lower than in the as-prepared state. The equilibrium concentration of PBDT, $\mathrm{C}_{\mathrm{p}, \mathrm{e}}$ can be calculated by dividing $\mathrm{C}_{\mathrm{p}, 0}$ by the swelling ratio, $\mathrm{Q}$. By plotting $\mathrm{C}_{\mathrm{p}, \mathrm{e}}$ versus $\mathrm{C}_{\mathrm{p}, 0}$ in Figure $\mathbf{5 b}$, we are directly comparing the relationship between the PBDT concentration in the as-prepared state and the equilibrium state in these gels. Samples falling below the line representing a slope of 1 exhibit greater swelling in water, because the difference between asprepared and equilibrium swelling is greater. As the PBDT concentration increases, the swelling ratio decreases (symbols fall closer to the line). From this we can infer that increased PBDT concentration results in a stronger sacrificial network.


Figure 5. Influence of PBDT concentration. a) Swelling ratio versus as-prepared PBDT concentration. b) A plot comparing equilibrium and as-prepared concentration, $C_{p, e}$ versus $C_{p, 0}$, which demonstrates the relative swelling nature of each composition. As the PBDT concentration increases, swelling decreases. c) Stress-strain curves of PBDT/PAAm gels with different concentrations of PBDT in the hydrogel matrix. Colors correspond with the concentrations from a) and b). d) Relationship between Young's modulus of PBDT/PAAm gels and PBDT concentration, either $\mathrm{C}_{\mathrm{p}, 0}$ or $\mathrm{C}_{\mathrm{p}, \mathrm{e}}$. The symbols represent the average of 3-5 samples and in all cases error bars are smaller than the symbol size. Red vertical band represents the literature overlap concentration, $C_{\text {ref }}{ }^{*}$ of PBDT. Vertical dotted black and gray lines represent the apparent critical concentrations of PBDT in either the equilibrium condition $\mathrm{C}_{\mathrm{p}, \mathrm{e}^{*}}$ or as-prepared condition $\mathrm{C}_{\mathrm{p}, 0}{ }^{*}$, respectively. Dashed trend lines are drawn as guides for the eye. All samples were equilibrated in $0.15 \mathrm{M} \mathrm{ZrCl}_{2} \mathrm{O}$ solution. 


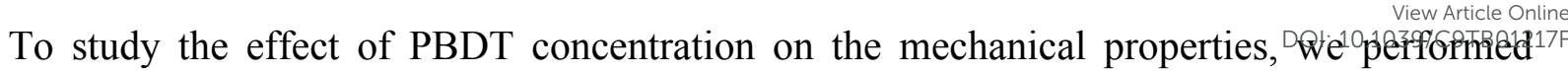
uniaxial tensile tests on the $\mathrm{ZrCl}_{2} \mathrm{O}$ solution-equilibrated PBDT/PAAm gels with different PBDT concentrations, Figure 5c. The colors of the plotted stress-strain curves match with the concentrations plotted in Figure 5a and Figure 5b. When only a small amount of PBDT is present $\left(C_{p, 0}=0.1 \mathrm{wt} \%\right)$, the sample exhibits properties similar to neat PAAm (plotted in black and almost entirely eclipsed). Increasing the PBDT content to $\mathrm{C}_{\mathrm{p}, 0}=1 \mathrm{wt} \%$ causes an increase in modulus, but a linear stress-strain response is maintained. Further increasing to $\mathrm{C}_{\mathrm{p}, 0}=2 \mathrm{wt} \%$ results in the introduction of a yielding point along with further increasing modulus. Young's modulus, yield stress, fracture strain and fracture stress continue to increase with increasing $\mathrm{C}_{\mathrm{p}, 0}$ up to $\mathrm{C}_{\mathrm{p}, 0}=5 \mathrm{wt} \%$, which possesses extremely high Young's modulus of $5.04 \pm 0.32 \mathrm{MPa}$, high fracture stress of $2.70 \pm 0.13 \mathrm{MPa}$, and high fracture strain of $10.8 \pm 0.15$. The enhancement ratio $\left(\mathrm{C}_{\mathrm{p}, 0}=5 \mathrm{wt} \%\right.$ PBDT / neat PAAm $)$ of Young's modulus and work of extension reached $\sim 280$ and $\sim 110$, respectively.

To understand what causes the transition to improved mechanical properties, Young's modulus is plotted both versus $C_{p, 0}$ and $C_{p, e}$ in Figure 5d. Two trends are clearly visible. At low concentration, the modulus increases slowly with increasing concentration, because PBDT acts merely as a filler and cannot form a network. At a critical concentration of $\mathrm{C}_{\mathrm{p}, 0}{ }^{*} \sim 0.8 \mathrm{wt} \%$ based on the asprepared state or $\mathrm{C}_{\mathrm{p}, \mathrm{e}}{ }^{*} \sim 0.3 \mathrm{wt} \%$ for the equilibrium state, a notable change in slope occurs, and modulus increases at a much quicker rate. $\mathrm{C}_{\mathrm{p}, 0}{ }^{*}$ is much higher than the reported overlap concentration, $\mathrm{C}_{\mathrm{ref}}{ }^{*}\left(\right.$ red band in Figure 5d), while $\mathrm{C}_{\mathrm{p}, \mathrm{e}}{ }^{*}$ matches the literature value of $\mathrm{C}_{\mathrm{ref}}{ }^{*}=0.3 \mathrm{wt} \%$, for PBDT with comparable molecular weight $\left(\mathrm{M}_{\mathrm{w}}=180 \mathrm{kDa}\right.$ versus $142 \mathrm{kDa}$ of this work $) .{ }^{14}$ This is reasonable since the ionic crosslinking occurs during the swelling process. For gels formed with $\mathrm{C}_{\mathrm{p}, 0}=0.6 \%$, in the as-prepared state the PBDT will overlap, but upon immersion in salt solution swelling occurs, resulting in $\mathrm{C}_{\mathrm{p}, \mathrm{e}}=0.2 \%$, and therefore a network structure cannot form. This sample exhibits low Young's modulus and does not undergo yielding. When the concentration of PBDT satisfies both overlap and possesses multi-valent crosslinking, a network forms that significantly increases the mechanical strength and toughness of the composite. These results indicate that only a few wt $\%$ of 


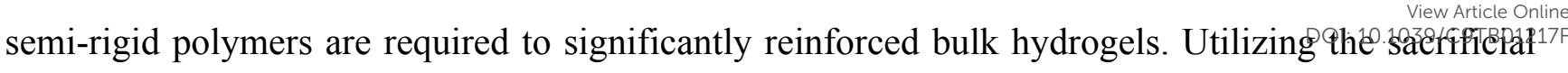
bond principle enabled highly effective enhancement of an ordinary hydrogel.

While hydrogels are generally clear, we noticed that large loadings of PBDT influenced the clarity of the samples. To quantify this change, we measured the transparency of the samples. The results are presented in Table S1. For the PAAm-ion samples, and for the PBDT/PAAm samples in water, the transparency was nearly $100 \%$. However, we see in the case of PBDT/PAAm in $\mathrm{ZrCl}_{2} \mathrm{O}$ solution, as the PBDT concentration increases, the transparency decreases. When $\mathrm{C}_{\mathrm{p}, \mathrm{e}}=0.39 \mathrm{wt} \%$, or just greater than $\mathrm{C}_{\mathrm{ref}}{ }^{*}$, transparency was $91 \%$. Increasing to $\mathrm{C}_{\mathrm{p}, \mathrm{e}}=1.8 \mathrm{wt} \%$ the transparency was $83 \%$. Further increasing to $\mathrm{C}_{\mathrm{p}, \mathrm{e}}=3.8 \mathrm{wt} \%$ and the transparency drops to $62 \%$. PBDT of similar molecular weight was shown to exhibit a critical nematic liquid crystal concentration, $\mathrm{C}_{\mathrm{LC}}{ }^{*}=2.2 \mathrm{wt} \%{ }^{18}$ Ordering within the gel likely contributes to both the increased mechanical response as well as the decreased transparency. In general, the formation of a physical sacrificial network with PBDT by $\mathrm{ZrCl}_{2} \mathrm{O}$ crosslinking results in large aggregates that are capable of scattering light. As these aggregates become stronger, the tensile properties increase, but the larger aggregates also scatter more light, resulting in a decrease in transparency. Based on these results, optimum physical DN gels are formed when the $\mathrm{C}_{\mathrm{p}, \mathrm{e}}$ is between $0.3 \mathrm{wt} \%$ and $2.2 \mathrm{wt} \%$, allowing high mechanical performance and maintaining clarity.

\section{Importance of Chain Rigidity}

Polymer chain rigidity is an important factor for creating sacrificial networks based on polyelectrolytes. For a polyelectrolyte with an intrinsically flexible chain, the charged backbone of the polyelectrolyte extends in pure water but changes to a coiled conformation in ionic solutions and even collapses in multi-valent ion solutions. We overcame this problem by using intrinsically rigid polyelectrolytes that can maintain their extended conformation even in high ionic strength environments. To test the importance of persistence length, we substituted PBDT with a relatively flexible polymer, poly(2-acrylamido-2-methyl propane sulfonic acid) sodium salt (PNaAMPS). In the presence of even small concentrations of salt, the persistence length of PNaAMPS is on the order of 


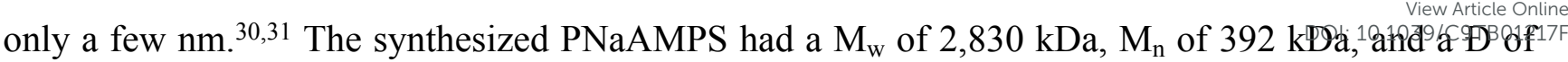
7.2. With such a large molecular weight, the $3 \mathrm{wt} \%$ PNaAMPS solution will be above the overlap concentration even in high ionic strength. After synthesizing a PAAm gel containing $3 \mathrm{wt} \%$ linear PNaAMPS, we immersed the PNaAMPS/PAAm gel in $0.15 \mathrm{M} \mathrm{Z} \mathrm{ZCl}_{2} \mathrm{O}$ solution, following the same method as for the PBDT/PAAm gels (see Supporting Information for details). After reaching the equilibrium state, we performed uniaxial tensile tests (Figure S1, Supporting Information). The PNaAMPS/PAAm gel was soft and elastic with low Young's modulus of $29 \pm 1.6 \mathrm{kPa}$, low fracture stress of $135 \pm 20 \mathrm{kPa}$, and fracture strain of $4.52 \pm 0.42$, contrasting the results seen for the PBDT/PAAm gel. Due to its high molecular weight, the PNaAMPS is expected to overlap and form a network. However, this physical network could not serve as a sacrificial network, because the presence of ions prevents chain extension. Without an extended network, the PNaAMPS chains do not break preferentially during deformation, and therefore a double network structure is not formed. The PBDT structure we use is inherently rigid, allowing it to work well to form an extended chain sacrificial network, even in the presence of ions. These results demonstrate that the rigidity of the physical gel polymer plays an important role, matching the essence of the extended polyelectrolyte $1^{\text {st }}$ network in conventional DN gel. ${ }^{[9]}$

\section{CONCLUSION}

The mechanical properties of semi-rigid PBDT / polyacrylamide composite hydrogels were significantly enhanced via swelling in $\mathrm{ZrCl}_{2} \mathrm{O}$ multi-valent ion solution. Toughening was induced by the creation of a stiff, physical sacrificial network; thus, the intensity of the cationic nature of the ions, the concentration of ions, the concentration of the semi-rigid polymers, and the rigidity of the polymer chains are all important parameters influencing the effectiveness of the sacrificial network. These networks possess partial self-healing ability. Along with these excellent properties, these hydrogels still contain $80 \%$ water. The strategy introduced here represents a useful method to create DN gels 


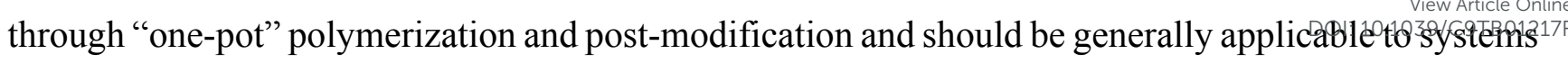
beyond PBDT/PAAm, including other rigid / stretchable polymer system such as rigid biopolymers (e.g. DNA, microtubule, and collagen) ${ }^{32}$ and flexible biopolymers (e.g. polysaccharides).$^{33}$ We believe that this method will find application as tunable soft materials for use in aqueous environments.

\section{EXPERIMENTAL SECTION}

Preparation of PBDT / PAAm hydrogels: To synthesize the hydrogel films, reaction cells were prepared by sandwiching a square framed silicone spacer (thickness: $1 \mathrm{~mm}$ ) between two parallel glass plates. The aqueous pre-gel solutions contained $3.0 \mathrm{M}$ of the neutral monomer, AAm, $0-5.0 \mathrm{wt} \%$ of the semi-rigid anionic polymer, PBDT $\left(\mathrm{M}_{\mathrm{w}}=142 \mathrm{kDa} ; \mathrm{M}_{\mathrm{n}}=110 \mathrm{kDa} ; \mathrm{P}=1.29\right), 0.1 \mathrm{~mol} \%$ of the chemical crosslinker, $N, N^{\prime}$-Methylenebisacrylamide (MBAA), and $0.5 \mathrm{~mol} \%$ of the thermal-initiator, potassium persulfate (KPS) (mol\% is relative to the monomer). Additional information about synthesis of PBDT can be found in the supporting information. After proper mixing, the pre-gel solution was poured into the reaction cell. Thermal-induced free radical polymerization was carried out in air in a temperature-controlled heat box $\left(60^{\circ} \mathrm{C}\right)$ for $10 \mathrm{~h}$. After thermal polymerization, the as-prepared sheetlike gels (about $60 \times 60 \times 1.0 \mathrm{~mm}^{3}$ ) were carefully removed from the reaction cells.

Swelling in various solutions containing multi-valent ions with pre-stretching: The as-prepared PBDT/PAAm gels were immersed in a large amount of pure water or different multi-valent ion solutions $\left(\mathrm{NaCl}, \mathrm{CaCl}_{2}, \mathrm{AlCl}_{3}, \mathrm{ZrCl}_{2} \mathrm{O}\right)$ for $24 \mathrm{~h}$, respectively. Unless otherwise stated, the concentration of the ionic solution was $0.15 \mathrm{M}$. Subsequently, the ion-loaded hydrogels were immersed in deionized water for $24 \mathrm{~h}$ to remove excess ions. The swelling ratio, $\mathrm{Q}$, or volume-change relative to the as-prepared state, $\mathrm{V} / \mathrm{V}_{0}$, was calculated from the size change in the three dimensions.

Mechanical testing: Uniaxial tensile tests were performed on swollen samples using a tensile- 
compressive tester (Instron 5965 type universal testing system). Samples were cut infó $19 \cdot \frac{\text { View Article Online }}{2}$ shape standardized as JIS-K6251-7 sizes (length $35 \mathrm{~mm}$, width $6 \mathrm{~mm}$, thickness 1.5-3, gauge length $12 \mathrm{~mm}$, inner width $2 \mathrm{~mm}$ ) with a gel cutting machine (Dumb Bell Co., Ltd). The fracture stress and fracture strain were defined as the nominal stress and strain at the breaking point, respectively. Young's modulus was defined as the initial slope of the stress-strain curves. Strain energy density was defined as the area under the stress-strain curve.

Cyclic loading/unloading tests: The cyclic loading/unloading tensile tests for evaluating the selfhealing ability of the PBDT/PAAm gels swollen in $\mathrm{ZrCl}_{2} \mathrm{O}$ solution was performed on one sample repeatedly by using an Instron 5965 type universal testing system. The PBDT/PAAm gels were stretched to $\varepsilon=0.5$ or 4 at a velocity of $100 \mathrm{~mm} / \mathrm{min}$ at room temperature. Then, samples were returned to the initial displacement immediately at the same velocity as stretching. After each loading / unloading test, the samples were left to stand at room temperature for a prescribed time $(1,5,30$ or 60 min). During testing, the samples were covered with silicone oil to prevent drying. The energy dissipation was estimated from the hysteresis area, $\mathrm{U}_{\text {hys }}$ by:

$$
U_{\text {hys }}=\int_{\varepsilon=0}^{\varepsilon=4}\left(\sigma_{\text {load }}-\sigma_{\text {unload }}\right) d \varepsilon
$$

Where $\sigma_{\text {load }}$ and $\sigma_{\text {unload }}$ are the stress during loading and unloading, respectively. From the $\mathrm{U}_{\text {hys }}$ of the virgin sample compared to subsequent tests after various waiting times, the healing efficiency (\%) was calculated.

Additional experimental details are described in Supporting Information.

\section{CONFLICTS OF INTEREST STATEMENT}

There are no conflicts to declare. 
This research was financially supported by Grant-in-Aid for Scientific Research (No. JP17H06144) and Grant-in-Aid for JSPS Fellows (No. 15J01078) from the Japan Society for the Promotion of Science (JSPS). R.T. was supported by MEXT through the Program for Leading Graduate Schools (Hokkaido University “Ambitious Leader’s Program”). Institute for Chemical Reaction Design and Discovery (ICReDD) was established by World Premier International Research Initiative (WPI), MEXT, Japan.

\section{REFERENCES}

1 S. Ma, M. Scaraggi, D. Wang, X. Wang, Y. Liang, W. Liu, D. Dini and F. Zhou, Adv. Funct. Mater., 2015, 25, 7366-7374.

2 P. Li, Y. F. Poon, W. Li, H.-Y. Zhu, S. H. Yeap, Y. Cao, X. Qi, C. Zhou, M. Lamrani, R. W. Beuerman, E.-T. Kang, Y. Mu, C. M. Li, M. W. Chang, S. S. Jan Leong and M. B. Chan-Park, Nat. Mater., 2011, 10, 149-156.

3 M. C. Branco, D. J. Pochan, N. J. Wagner and J. P. Schneider, Biomaterials, 2010, 31, 95279534.

4 M. Shibayama, Bull. Chem. Soc. Jpn., 2006, 79, 1799-1819.

$5 \quad$ K. S. Anseth, C. N. Bowman and L. Brannon-Peppas, Biomaterials, 1996, 17, 1647-1657.

6 Y. Okumura and K. Ito, Adv. Mater., 2001, 13, 485-487.

7 N. Sasaki, T. Matsunaga, M. Shibayama, S. Suzuki, Y. Yamamoto, R. Yoshida, C. Ito, T. Sakai and U. Chung, Macromolecules, 2008, 41, 5379-5384.

$8 \quad$ K. Haraguchi and T. Takehisa, Adv. Mater., 2002, 14, 1120.

9 J. P. Gong, Y. Katsuyama, T. Kurokawa and Y. Osada, Adv. Mater., 2003, 15, 1155-1158.

10 T. Nakajima, H. Furukawa, Y. Tanaka, T. Kurokawa, Y. Osada and J. P. Gong, Macromolecules, 2009, 42, 2184-2189.

11 J. P. Gong, Soft Matter, 2010, 6, 2583.

12 J. Saito, H. Furukawa, T. Kurokawa, R. Kuwabara, S. Kuroda, J. Hu, Y. Tanaka, J. P. Gong, N. Kitamura and K. Yasuda, Polym. Chem., 2011, 2, 575-580.

13 K. Yasuda, N. Kitamura, J. P. Gong, K. Arakaki, H. J. Kwon, S. Onodera, Y. M. Chen, T. Kurokawa, F. Kanaya, Y. Ohmiya and Y. Osada, Macromol. Biosci., 2009, 9, 307-316.

14 W. Yang, H. Furukawa, Y. Shigekura, K. Shikinaka, Y. Osada and J. P. Gong, Macromolecules, 
2008, 41, 1791-1799.

15 N. Sarkar and L. D. Kershner, J. Appl. Polym. Sci., 1996, 62, 393-408.

16 Y. Wang, J. Gao, T. J. Dingemans and L. A. Madsen, Macromolecules, 2014, 47, 2984-2992.

17 Y. Wang, Y. Chen, J. Gao, H. G. Yoon, L. Jin, M. Forsyth, T. J. Dingemans and L. A. Madsen, Adv. Mater., 2016, 28, 2571-2578.

18 Z. L. Wu, T. Kurokawa, S. Liang, H. Furukawa and J. P. Gong, J. Am. Chem. Soc., 2010, 132, 10064-10069.

19 W. Yang, H. Furukawa and J. P. Gong, Adv. Mater., 2008, 20, 4499-4503.

20 Z. L. Wu, T. Kurokawa, D. Sawada, J. Hu, H. Furukawa and J. P. Gong, Macromolecules, 2011, 44, 3535-3541.

21 C. H. Yang, M. X. Wang, H. Haider, J. H. Yang, J. Y. Sun, Y. M. Chen, J. Zhou and Z. Suo, ACS Appl. Mater. Interfaces, 2013, 5, 10418-10422.

22 T. Nakajima, H. Sato, Y. Zhao, S. Kawahara, T. Kurokawa, K. Sugahara and J. P. Gong, Adv. Funct. Mater., 2012, 22, 4426-4432.

23 K. Matsui and M. Ohgai, J. Am. Ceram. Soc., 2005, 80, 1949-1956.

24 T. Matsuda, T. Nakajima, Y. Fukuda, W. Hong, T. Sakai, T. Kurokawa, U. Il Chung and J. P. Gong, Macromolecules, 2016, 49, 1865-1872.

25 J.-Y. Sun, X. Zhao, W. R. K. Illeperuma, O. Chaudhuri, K. H. Oh, D. J. Mooney, J. J. Vlassak and Z. Suo, Nature, 2012, 489, 133-6.

26 T. L. Sun, T. Kurokawa, S. Kuroda, A. Bin Ihsan, T. Akasaki, K. Sato, M. A. Haque, T. Nakajima and J. P. Gong, Nat. Mater., 2013, 12, 932-937.

27 P. Lin, S. Ma, X. Wang and F. Zhou, Adv. Mater., 2015, 27, 2054-2059.

28 K. Sato, T. Nakajima, T. Hisamatsu, T. Nonoyama, T. Kurokawa and J. P. Gong, Adv. Mater., 2015, 27, 6990-6998.

29 H. J. Zhang, T. L. Sun, A. K. Zhang, Y. Ikura, T. Nakajima, T. Nonoyama, T. Kurokawa, O. Ito, H. Ishitobi and J. P. Gong, Adv. Mater., 2016, 28, 4884-4890.

30 M. Tricot, Macromolecules, 1984, 17, 1698-1704.

31 T. Hattori, S. Bat-Aldar, R. Kato, H. B. Bohidar and P. L. Dubin, Anal. Biochem., 2005, 342, 229-236.

32 P. A. Janmey, D. R. Slochower, Y. H. Wang, Q. Wen and A. Cebers, Soft Matter, 2014, 10, 1439-1449.

33 R. L. Cleland, Arch. Biochem. Biophys., 1977, 180, 57-68. 